\title{
ASYMMETRIC PROPERTIES OF HEART RATE VARIABILITY TO ASSESS OPERATOR FATIGUE
}

\author{
Christian Heinze ${ }^{1}$, Udo Trutschel ${ }^{2}$, Dave Edwards ${ }^{3}$, Bill Sirois ${ }^{2}$, \& Martin Golz ${ }^{1}$ \\ ${ }^{1}$ Faculty of Computer Science, University of Applied Sciences Schmalkalden, Germany \\ ${ }^{2}$ Circadian Technologies, Inc., Stoneham, Massachusetts, USA \\ ${ }^{3}$ Caterpillar, Inc., Peoria, Illinois, USA
}

\begin{abstract}
Summary: The aim of this study is to evaluate the suitability of heart rate recordings for establishing a reliable connection to well-defined fatigue and performance measures in order to estimate fatigue in industrial and transportation applications. An overnight driving simulation scenario with partial sleep deprivation was utilized to induce strong fatigue. An experiment trial was divided into repeated sessions, each of which consisted of a driving performance and two vigilance tasks. Heart rate (HR) was recorded over the entire experiment; HRmeasures were derived and correlated against measures that were established from driving and vigilance task performance and that represent various aspects of operator fatigue. In a previous report (Hefner et al. 2009) we presented on the basis of the data of one volunteer that multiple fatigue measures correlate well with different expressions of heart rate variability (HRV), especially with longterm HRV derived from Poincaré plots. In this work, we intensify the Poincaré analysis by dividing the distribution of HR data in different accelerating and decelerating segments and by establishing properties of asymmetry between these segments. We also show that most of the properties of long-term HRV correlate well with specific fatigue measures for a group of 5 volunteers despite their large inter-individual differences in HR-to-fatigue correlations.
\end{abstract}

\section{INTRODUCTION}

Worker fatigue is a major concern for any operation that requires sustained employee alertness and performance, especially in industries where 24/7 shift work schedules are utilized, such as in mining, transportation and emergency services. During task performance, subjects have to expend physical and mental effort, which is usually reflected in increased heart rate (HR, number of heart beats in a certain time period) and decreased heart rate variability (HRV, phenomenon of varying time durations between heart beats, expression of balance between sympathetic and parasympathetic branch of autonomous nervous system) when compared to resting situations. In contrast to this, in a state of fatigue or relaxation HR will decrease and HRV will increase. Therefore, during periods where both workload and fatigue are high, HR measures can expose contradicting sensitivities. To add to this complexity, Coumel et al. (1995) pointed out that several HR measures show a strong circadian behavior under normal sleeping conditions. Therefore, a correlation to fatigue can be expected. As a consequence, the behavior of HR measures in general, and their ability to indicate fatigue in particular, can only be established under strict experimental conditions eliminating the influence of mental workload. In this experimental protocol of driving and testing (CTT and PVT), the mental workload and their influences were reduced to a minimum. 


\section{DATA COLLECTION}

The study was conducted at the Department of Adaptive Bio-Signal Analysis at the University of Applied Sciences, Schmalkalden (Germany). In each trial, a volunteer had to accomplish a series of driving simulations. The driving situation was laid out for monotony in order to catalyze fatigue: the task was to simply hold the lane on a road without intersections, crossroads or any other cars present through a landscape at dark night at high speed. Before a trial, a volunteer carried an activity monitor and completed a sleep/wake log for at least 24 hours prior to the trial. A volunteer arrived at the lab at 10:00 pm. After wire-up, checking logs and activity monitors, experiment sessions started at 11:30 pm. There were eight experiment sessions in one trial, each lasting one hour, thus the last session finished at 8:30 am. Volunteers had a 1-hour break at 3:30 am. Each session included a 40-minute driving task, a 10-minute Compensatory Tracking Task (CTT), and a 5-minute Psychomotoric Vigilance Test (PVT). A total of 5 volunteers participated in the study, each whom completed two separate trials.

\section{DATA ANALYSIS}

\section{Fatigue Measures}

Driving Sessions. During each 40-minute driving session (eight driving sessions per trial), the following parameter set (fatigue measure: definitions in italics, (labels) in parenthesis) allowed to capture the different facets of driver fatigue:

- Typical signs of micro-sleep events (MSE) are prolonged eyelid closures, rolling eye movements, head nodding, and stare gaze. MSE are an objective, individually specific fatigue measure. The occurrence and severity of MSE was visually scored and logged during driving, resulting in a weighted sum of MSE per driving session.

- Lateral lane deviation is a continuously recorded measure of driving performance. The standard deviation of lateral lane deviation (LaneDev) over an entire driving session forms another objective.

- Accident events represent the consequences of the inability to stay awake and keep the lane. The measure (Acc) is the number of accident events over an entire driving session.

- The Karolinska Sleepiness Scale is a standardized, subjective and independent measure of fatigue on a numeric scale between 1 and 10, which the volunteer stated verbally every four minutes during driving. Those values were averaged per driving session (KSS).

- Percentage of eyelid closure, or PERCLOS - promoted for many years as an excellent objective measure of fatigue - was continuously recorded by a commercially available eye-tracking system. The average of PERCLOS over a driving session is called (PERCL).

CTT Sessions. The Compensatory Tracking Task (Van Orden et al. 2000) is a two-dimensional visual-motoric performance task involving a target positioned in the center of a display and a moving disk. The task is to keep the disk as near to the center of the target annulus as possible, using a trackball whose movement induces a restorative force to the disk in the direction of the trackball motion. The task was performed for 10 minutes. The position of the disk is recorded as a function of its previous position and velocity. The mean and standard deviation of distance of the moving disk to the target (M/SD Dist), the mean and standard deviation of velocity of the 
disk (M/SD Velc) and the mean and standard deviation of force applied to the disk (M/SD Force) are used as set of CTT fatigue measures.

PVT Sessions. The Psychomotoric Vigilance Test (PVT) is a reaction task: the subject is instructed to respond to a visual stimulus (that is given in random intervals) with a button-push as quickly as possible. The PVT used in our study was the short 5-minute version evaluated in Lamond et al. (2005). Latency is defined as the time duration between the appearance of the stimulus and the response. The mean and standard deviation of latency (M/SD Latc) as well as the number of lapses, i.e. the absence of a response, were selected as set of PVT fatigue measures.

\section{Heart Rate Measures}

The position of R-peaks (i.e. heart beats) was detected in electrocardiogram (ECG) recordings. Calculating the time interval between consecutive beats results in an RR-interval time series with characteristic patterns and frequency contents. Artifacts or variant beats were excluded or corrected, respectively, by a thorough visual inspection of each series.

Time Domain. For a segment of RR intervals - each during driving, CTT and PVT - the simplest measures are its mean and standard deviation, which we denote as heart rate (HR) and heart rate variability (HRV), respectively. All consecutive RR intervals differing more than 50 milliseconds were counted and normalized by the length of the RR intervals segment, resulting in the measure (pNN50). After z-transforming the RR series, its zero-crossings were counted and normalized as well (ZeroCross).

Phase Space. Levy (1977) showed a clear nonlinear relation between sympathetic and parasympathetic activity at one side and HR at the other. A simple method to capture this nonlinear characteristic is the so-called Poincaré plot in phase space. It is both a useful visual tool which can summarize an entire RR series and a quantitative technique. A Poincaré plot of RR intervals is composed of points $\left(R R_{i}, R R_{i+1}\right)$, that is each point in the plot corresponds to two consecutive RR intervals. The resulting cloud of points (see Figure 1) is usually characterized by two common descriptors: its length along the line of identity (SD2, describes long-term HRV) and its breadth across this line (SD1, describes short-term HRV). In most Poincaré plots of RR intervals, there is a visible and statistically highly significant asymmetry (Piskorski \& Guzik 2007), with the upper part (decelerations) larger than the lower part (accelerations). In order to quantify those asymmetries, the Poincaré data distribution is transformed into Cartesian coordinates and then divided into four quadrants. In each quadrant, short- and long-term variations are calculated, resulting in the following 8 local descriptors (see Figure 2):

- Acceleration along major axis (SD1a_right \& SD2a_right)

- Acceleration along minor axis (SD1a_low \& SD2a_low)

- Deceleration along major axis (SD1d_left \& SD2d_left)

- Deceleration along minor axis (SD1d_high \& SD2d_high)

To express asymmetries between the four quadrants, the following 4 asymmetry properties are established from the local descriptors: 
- Asymmetry ratios of opposite quadrants:

$S D 1 A S=\frac{S D 1 a_{-} \text {right }-S D 1 d_{-} \text {left }}{S D 1}, \quad S D 2 A S=\frac{S D 2 a_{-} l o w-S D 2 d_{-} \text {high }}{S D 2}$

- Asymmetry ratios of quadrants on the same side:

$S D 1 A S S=\frac{S D 1 a_{-} l o w-S D 1 d_{\_} \text {high }}{S D 1}, \quad S D 2 A S S=\frac{S D 2 a_{-} r i g h t-S D 2 d_{\_} l e f t}{S D 2}$

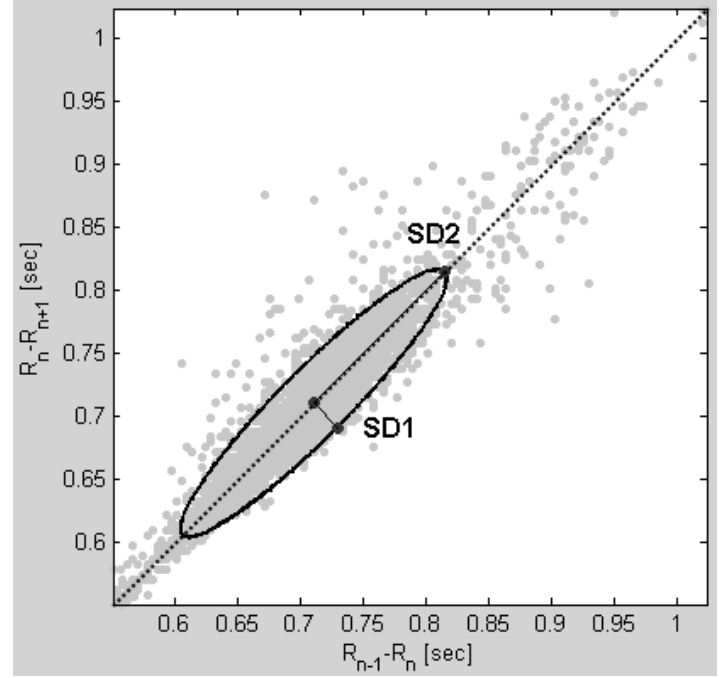

Figure 1. An example of Poincaré plot of RR intervals with fitted ellipse; (Diagonal dotted line, the "identity line" helps divide all points to decelerations or accelerations)

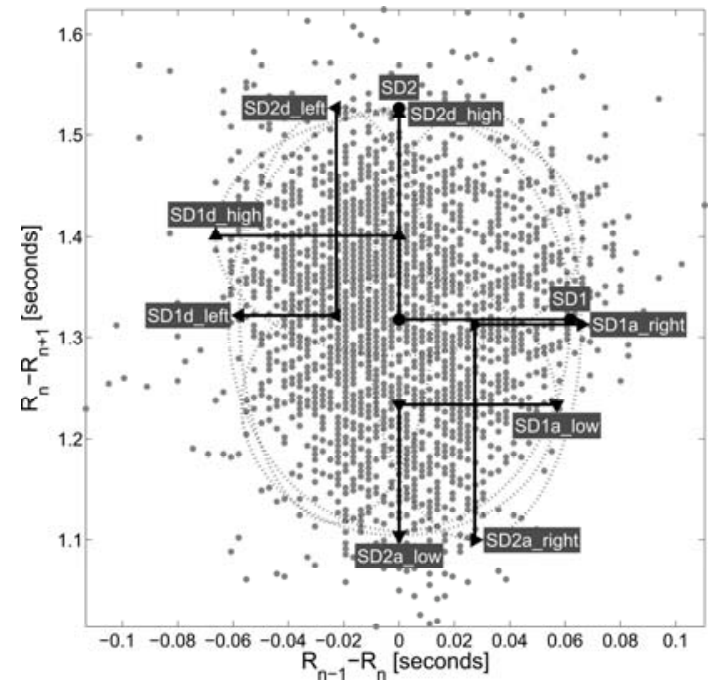

Figure 2. Poincaré plot of RR intervals during 40 minutes of driving session, in Cartesian coordinates with various asymmetric properties of short- \& long-term HRV

These asymmetric properties are also calculated without being normalized against the global descriptors SD1 \& SD2, resulting in the according asymmetries:

- SD1a_right-SD1d_left

- SD2a_low-SD2d_high

- SD1a_low-SD1d_high

- SD2a_right-SD2d_left

\section{Comparison of heart rate measures and fatigue measures}

Correlation coefficients between individual HR- and fatigue measures were computed. For each coefficient, session values of both trials (i.e. 16 sessions) of a HR and a fatigue measures were correlated. This approach averages (and therefore neglects) the intra-individual differences between both trials of each volunteer. In the next step, inter-individual differences were averaged in order to reveal universal coherences between HR- and fatigue measures. First, all session values (i.e. 16 sessions) of each measure of each subject were z-scored. Then, each session value of each measure was averaged over all 5 subjects. Finally, correlations were computed by pairing session values of each HR-measures with respective session values of each fatigue measure. Correlations were considered significant for p-values $<0.01$. 


\section{RESULTS}

As stated in our previous report (Hefner et al. 2009), the fatigue measures of driving, CTT and PVT generally show a clear time-on-task and a modest time-of-day behaviour: fatigue is low during first sessions and significantly higher in the second half of the trial. Maximum fatigue occurs during the third- or second-to-last session, between 5:30 and 7:30 pm. In order to correlate well with fatigue measures, the HR-measures would have to follow this course (or exactly vice versa, in case of negative correlation).

Tables 1-3 present a selection of individual correlation patterns. Inter-individual differences between the subjects are most obvious; the discrepancies are as extreme as for subjects 1 and 5 (Tables 1 and 3); where for the former almost a third of all fatigue and HR-measures correlate significantly, whereas for the latter no (except one) combination of HR- and fatigue measure exhibits significance. Table 4 reveals that the best coherence between HR- and fatigue measures is obtained for the asymmetry properties of long-term HRV (SD2[...]) and lateral lane deviation, respectively. Lane deviation and accident score generally show the same correlation patterns, since both are highly inter-dependent. Also, CTT and PVT measures, mainly distance and latency, respectively, correlate well with asymmetry properties of long-term HRV, even though in smaller number. None of the asymmetry ratios of long-term HRV correlates with any fatigue measures. Also, one exception aside, there's no correlation between measures of short-term HRV (SD1[...]) and fatigue. In the time domain, two different expressions of heart rate variability, standard deviation (HRV) and ZeroCross, show the same good correlation behaviour; mean heart rate (HR) and pNN50, on the other hand, are insignificant. Overall, the HR-measure ZeroCross exhibits the best coherence with fatigue measures.

\section{CONCLUSIONS}

The analysis of asymmetric properties of HRV confirms that long-term HRV in general correlates well with selected fatigue measures, but it didn't reveal further details as to where this link originates (e.g. from acceleration or deceleration of the heart rate). One the other hand, it is pleasant that a systematic coherence of long-term HRV to selected fatigue measures could be established from a group of subjects despite their huge inter-individual differences. For the CTT and PVT condition, the expectation that distance and latency, respectively, will relate well to HR-measures was supported. On the other hand, it is curious that for the driving condition only lateral lane deviation (and accident score, its close relative) exhibits good correlations to HRmeasures. Particularly MSE and KSS were expected to correlate well with HR-measures, since both have been proven to be reliable measures for fatigue (Sommer et al. 2009). A possible explanation might be that the time courses of MSE and KSS on one hand and of HR-measures on the other are linked by a nonlinear relationship that cannot be revealed by correlation analysis. The strong coherence between long-term HRV properties and specific fatigue measures suggests the possibility to predict driver and operator fatigue in the middle-term (around 30 minutes) solely from recordings of heart rate. Future analysis will show if these results will hold true for larger study populations. 
Table 1. Correlation coefficients between HR- and fatigue measures for subject 1

\begin{tabular}{|c|c|c|c|c|c|c|c|c|c|c|c|c|c|c|}
\hline \multirow[b]{2}{*}{$\mathrm{p}<0.01$} & \multicolumn{5}{|c|}{ Driving } & \multicolumn{6}{|c|}{ CTT } & \multicolumn{3}{|c|}{ PVT } \\
\hline & MSE & Acc & KSS & LaneDev & PERCL & M Dist & SD Dist & M Vele & SD Vele & M Force & SD Force & M Late & SD Late & Lapses \\
\hline HR & 0.5788 & 0.5495 & 0.6290 & 0.6213 & 0.2099 & 0.5466 & 0.4443 & 0.3778 & 0.2831 & -0.1851 & -0.0854 & 0.5466 & 0.4443 & 0.3778 \\
\hline HRV & 0.3378 & 0.7910 & 0.3399 & 0.8591 & 0.0010 & 0.7463 & 0.7043 & 0.7177 & 0.6430 & 0.3647 & 0.4167 & 0.7463 & 0.7043 & 0.7177 \\
\hline pNN50 & 0.2319 & 0.6472 & 0.3997 & 0.7204 & -0.1009 & 0.6883 & 0.5789 & 0.5217 & 0.3908 & -0.0158 & 0.0577 & 0.6883 & 0.5789 & 0.5217 \\
\hline ZeroCross & -0.3131 & -0.6874 & -0.3157 & -0.7530 & 0.0303 & 0.0811 & 0.0661 & -0.0616 & -0.0701 & -0.3989 & -0.2976 & 0.0811 & 0.0661 & -0.0616 \\
\hline SD1 & 0.3245 & 0.7387 & 0.4715 & 0.7222 & 0.0152 & 0.7729 & 0.6710 & 0.5926 & 0.4534 & -0.0705 & 0.0490 & 0.7729 & 0.6710 & 0.5926 \\
\hline SD1a_right & 0.4648 & 0.7712 & 0.4803 & 0.7041 & 0.0820 & 0.7854 & 0.6938 & 0.6172 & 0.4771 & -0.0398 & 0.0665 & 0.7854 & 0.6938 & 0.6172 \\
\hline SD1d_left & 0.2852 & 0.7058 & 0.4442 & 0.6473 & 0.1069 & 0.7854 & 0.7112 & 0.6500 & 0.5276 & 0.0457 & 0.1486 & 0.7854 & 0.7112 & 0.6500 \\
\hline SD1a_low & 0.3035 & 0.6117 & 0.4051 & 0.7327 & 0.0374 & 0.4797 & 0.3555 & 0.3375 & 0.2270 & -0.1170 & -0.0799 & 0.4797 & 0.3555 & 0.3375 \\
\hline SD1d_high & 0.3782 & 0.6902 & 0.5316 & 0.6226 & 0.1398 & 0.8185 & 0.7506 & 0.6710 & 0.5468 & 0.0443 & 0.1636 & 0.8185 & 0.7506 & 0.6710 \\
\hline SD1AS & 0.2517 & -0.0027 & 0.0792 & 0.0398 & 0.0069 & -0.0647 & -0.1246 & -0.1157 & -0.1857 & -0.1896 & -0.1762 & -0.0647 & -0.1246 & -0.1157 \\
\hline SD1ASS & -0.4610 & -0.2538 & -0.3506 & -0.0875 & -0.5523 & -0.7154 & -0.7779 & -0.7244 & -0.7116 & -0.4468 & -0.5294 & -0.7154 & -0.7779 & -0.7244 \\
\hline SD2 & 0.3383 & 0.7861 & 0.3336 & 0.8565 & 0.0012 & 0.7301 & 0.6909 & 0.7095 & 0.6397 & 0.3809 & 0.4284 & 0.7301 & 0.6909 & 0.7095 \\
\hline SD2a_right & 0.3565 & 0.8069 & 0.3373 & 0.8651 & 0.0173 & 0.7416 & 0.7015 & 0.7162 & 0.6492 & 0.3711 & 0.4243 & 0.7416 & 0.7015 & 0.7162 \\
\hline SD2d_left & 0.3212 & 0.7644 & 0.3295 & 0.8436 & -0.0099 & 0.7127 & 0.6756 & 0.6975 & 0.6248 & 0.3936 & 0.4329 & 0.7127 & 0.6756 & 0.6975 \\
\hline SD2a_low & 0.2550 & 0.7593 & 0.2268 & 0.8420 & -0.1196 & 0.7047 & 0.6606 & 0.6458 & 0.5851 & 0.2700 & 0.3329 & 0.7047 & 0.6606 & 0.6458 \\
\hline SD2d_high & 0.5596 & 0.6606 & 0.3935 & 0.6956 & 0.3053 & 0.6948 & 0.6942 & 0.7054 & 0.6725 & 0.4354 & 0.4776 & 0.6948 & 0.6942 & 0.7054 \\
\hline SD2AS & -0.2855 & -0.0001 & -0.1746 & 0.1390 & -0.4556 & -0.0721 & -0.1315 & -0.1753 & -0.2209 & -0.3121 & -0.2812 & -0.0721 & -0.1315 & -0.1753 \\
\hline SD2ASS & 0.3572 & 0.2995 & 0.1832 & 0.1860 & 0.2883 & 0.4888 & 0.4568 & 0.4255 & 0.4317 & 0.0868 & 0.1975 & 0.4888 & 0.4568 & 0.4255 \\
\hline SD1a_right-SD1d_left & 0.1401 & -0.1534 & -0.0704 & -0.1263 & -0.0492 & -0.2774 & -0.2952 & -0.2850 & -0.2904 & -0.1657 & -0.2196 & -0.2774 & -0.2952 & -0.2850 \\
\hline SD2a_low-SD2d_high & -0.1157 & 0.1943 & -0.0404 & 0.3588 & -0.3418 & -0.0113 & -0.0529 & -0.0770 & -0.1065 & -0.1587 & -0.1720 & -0.0113 & -0.0529 & -0.0770 \\
\hline SD1a_low-SD1d_high & -0.4621 & -0.2630 & -0.3597 & -0.1007 & -0.5548 & -0.7484 & -0.7849 & -0.7110 & -0.6681 & -0.3255 & -0.4326 & -0.7484 & -0.7849 & -0.7110 \\
\hline SD2a_right-SD2d_left & 0.4683 & 0.4626 & 0.2911 & 0.3256 & 0.3197 & 0.6409 & 0.5848 & 0.5411 & 0.5026 & 0.0707 & 0.2057 & 0.6409 & 0.5848 & 0.5411 \\
\hline
\end{tabular}

Table 2. Correlation coefficients between HR- and fatigue measures for subject 3

\begin{tabular}{|c|c|c|c|c|c|c|c|c|c|c|c|c|c|c|}
\hline \multirow[b]{2}{*}{$\mathrm{p}<0.01$} & \multicolumn{5}{|c|}{ Driving } & \multicolumn{6}{|c|}{ CTT } & \multicolumn{3}{|c|}{ PVT } \\
\hline & MSE & Ace & KSS & LaneDev & PERCL & M Dist & SD Dist & M Velc & SD Vele & M Force & SD Force & M Late & SD Late & Lapses \\
\hline HR & -0.0103 & 0.0012 & 0.1398 & 0.0225 & 0.0100 & 0.5481 & 0.5767 & 0.0118 & -0.3032 & -0.5239 & -0.4060 & 0.5481 & 0.5767 & 0.0118 \\
\hline HRV & 0.4843 & 0.6152 & 0.6576 & 0.6846 & 0.1932 & 0.4509 & 0.5926 & 0.3183 & 0.2109 & -0.2422 & 0.0983 & 0.4509 & 0.5926 & 0.3183 \\
\hline pNN50 & 0.3743 & 0.4269 & 0.4272 & 0.4939 & 0.1047 & 0.8055 & 0.8520 & 0.4282 & 0.1256 & -0.2592 & 0.0306 & 0.8055 & 0.8520 & 0.4282 \\
\hline ZeroCross & -0.4096 & -0.5552 & -0.6933 & -0.6284 & -0.2028 & 0.3538 & 0.2794 & 0.0139 & -0.1659 & -0.0127 & -0.1461 & 0.3538 & 0.2794 & 0.0139 \\
\hline SD1 & 0.4417 & 0.4907 & 0.5160 & 0.5736 & 0.0918 & 0.7199 & 0.7706 & 0.3356 & 0.1198 & -0.2512 & 0.0457 & 0.7199 & 0.7706 & 0.3356 \\
\hline SD1a_right & 0.7047 & 0.7147 & 0.7241 & 0.7537 & 0.3212 & 0.7245 & 0.8037 & 0.4006 & 0.2188 & -0.1527 & 0.2164 & 0.7245 & 0.8037 & 0.4006 \\
\hline SD1d_left & 0.2901 & 0.3357 & 0.3929 & 0.4398 & -0.0400 & 0.5681 & 0.6095 & 0.3045 & 0.1476 & -0.1670 & 0.0604 & 0.5681 & 0.6095 & 0.3045 \\
\hline SD1a_low & 0.3513 & 0.3922 & 0.3436 & 0.3976 & 0.0853 & 0.5822 & 0.6343 & 0.0150 & -0.2372 & -0.4899 & -0.3220 & 0.5822 & 0.6343 & 0.0150 \\
\hline SD1d_high & 0.4581 & 0.4863 & 0.5887 & 0.6179 & 0.0827 & 0.5334 & 0.5713 & 0.5061 & 0.4261 & 0.1027 & 0.4038 & 0.5334 & 0.5713 & 0.5061 \\
\hline SD1AS & 0.6332 & 0.5936 & 0.5244 & 0.5063 & 0.5392 & 0.3455 & 0.3860 & 0.1955 & 0.1032 & -0.0289 & 0.2086 & 0.3455 & 0.3860 & 0.1955 \\
\hline SD1ASS & -0.1815 & -0.1234 & -0.3500 & -0.2617 & 0.0225 & 0.0505 & 0.0839 & -0.3733 & -0.5093 & -0.4777 & -0.5621 & 0.0505 & 0.0839 & -0.3733 \\
\hline SD2 & 0.4863 & 0.6257 & 0.6707 & 0.6933 & 0.2033 & 0.3984 & 0.5470 & 0.3116 & 0.2232 & -0.2332 & 0.1066 & 0.3984 & 0.5470 & 0.3116 \\
\hline SD2a_right & 0.4587 & 0.5990 & 0.6522 & 0.6619 & 0.1943 & 0.3931 & 0.5358 & 0.2923 & 0.1959 & -0.2601 & 0.0817 & 0.3931 & 0.5358 & 0.2923 \\
\hline SD2d_left & 0.5194 & 0.6563 & 0.6947 & 0.7281 & 0.2155 & 0.3999 & 0.5563 & 0.3259 & 0.2497 & -0.2013 & 0.1328 & 0.3999 & 0.5563 & 0.3259 \\
\hline SD2a_low & 0.4834 & 0.5679 & 0.5999 & 0.6088 & 0.1663 & 0.5765 & 0.6700 & 0.1382 & -0.0935 & -0.4944 & -0.2396 & 0.5765 & 0.6700 & 0.1382 \\
\hline SD2d_high & 0.5010 & 0.6375 & 0.6898 & 0.7147 & 0.2528 & 0.0821 & 0.2664 & 0.4474 & 0.5339 & 0.2241 & 0.4723 & 0.0821 & 0.2664 & 0.4474 \\
\hline SD2AS & -0.1941 & -0.2836 & -0.4387 & -0.3855 & -0.1871 & 0.5182 & 0.5326 & -0.1123 & -0.4255 & -0.5829 & -0.5222 & 0.5182 & 0.5326 & -0.1123 \\
\hline SD2ASS & -0.6716 & -0.6995 & -0.5549 & -0.7549 & -0.2110 & 0.0062 & -0.1266 & -0.0823 & -0.1000 & -0.3470 & -0.1578 & 0.0062 & -0.1266 & -0.0823 \\
\hline SD1a_right-SD1d_left & 0.4293 & 0.3838 & 0.3001 & 0.2704 & 0.4863 & 0.0810 & 0.1128 & 0.0651 & 0.0577 & 0.0648 & 0.1870 & 0.0810 & 0.1128 & 0.0651 \\
\hline SD2a_low-SD2d_high & -0.0663 & -0.1103 & -0.1892 & -0.1874 & -0.1045 & 0.5397 & 0.5960 & -0.0346 & -0.3140 & -0.5655 & -0.4359 & 0.5397 & 0.5960 & -0.0346 \\
\hline SD1a_low-SD1d_high & -0.1953 & -0.1505 & -0.3250 & -0.2913 & 0.0382 & 0.1141 & 0.1323 & -0.3768 & -0.5388 & -0.5557 & -0.6058 & 0.1141 & 0.1323 & -0.3768 \\
\hline SD2a_right-SD2d_left & -0.4713 & -0.4717 & -0.2354 & -0.5080 & -0.0810 & 0.0521 & -0.0642 & -0.0551 & -0.0794 & -0.3692 & -0.1464 & 0.0521 & -0.0642 & -0.0551 \\
\hline
\end{tabular}

Table 3. Correlation coefficients between HR- and fatigue measures for subject 5

\begin{tabular}{|c|c|c|c|c|c|c|c|c|c|c|c|c|c|c|}
\hline \multirow[b]{2}{*}{$p<0.01$} & \multicolumn{5}{|c|}{ Driving } & \multicolumn{6}{|c|}{ CTT } & \multicolumn{3}{|c|}{ PVT } \\
\hline & MSE & Acc & KSS & LaneDev & PERCL & M Dist & SD Dist & M Velc & SD Vele & M Force & SD Force & M Late & SD Late & Lapses \\
\hline HR & 0.2987 & 0.2821 & 0.2685 & 0.2678 & 0.3018 & 0.1789 & 0.1478 & $\begin{array}{l}-0.1337 \\
-0.13\end{array}$ & -0.2389 & -0.1969 & -0.2490 & 0.1789 & 0.1478 & -0.1337 \\
\hline HRV & 0.5049 & 0.5151 & 0.3480 & 0.5305 & 0.0716 & 0.2148 & 0.2291 & 0.0852 & 0.0788 & 0.0113 & 0.0686 & 0.2148 & 0.2291 & 0.0852 \\
\hline pNN50 & 0.1427 & 0.1448 & 0.0437 & 0.0730 & 0.2433 & 0.1211 & 0.1058 & -0.2738 & -0.1874 & -0.2991 & -0.1562 & 0.1211 & 0.1058 & -0.2738 \\
\hline ZeroCross & -0.3796 & -0.4020 & -0.4052 & -0.2715 & 0.0969 & -0.3408 & -0.3154 & -0.4297 & -0.3285 & -0.3167 & -0.2267 & -0.3408 & -0.3154 & -0.4297 \\
\hline SD1 & 0.2469 & 0.2617 & -0.0186 & 0.1005 & -0.0148 & 0.0817 & 0.0650 & -0.2220 & -0.2296 & -0.2870 & -0.2028 & 0.0817 & 0.0650 & -0.2220 \\
\hline SD1a_right & 0.3076 & 0.3278 & -0.0226 & 0.1607 & 0.0450 & 0.0922 & 0.1185 & -0.0616 & -0.1039 & -0.0896 & -0.0650 & 0.0922 & 0.1185 & -0.0616 \\
\hline SD1d_left & 0.2421 & 0.2612 & -0.1692 & 0.0689 & -0.3003 & -0.0557 & -0.0932 & -0.2903 & -0.3185 & -0.3481 & -0.2959 & -0.0557 & -0.0932 & -0.2903 \\
\hline SD1a_low & 0.1722 & 0.1908 & -0.0094 & 0.1273 & 0.1188 & 0.0483 & 0.0415 & -0.1733 & -0.2335 & -0.2409 & -0.2221 & 0.0483 & 0.0415 & -0.1733 \\
\hline SD1d_high & 0.2789 & 0.2897 & -0.0042 & 0.0794 & -0.0828 & 0.0705 & 0.0512 & -0.2450 & -0.2030 & -0.2908 & -0.1597 & 0.0705 & 0.0512 & -0.2450 \\
\hline SD1AS & -0.1268 & -0.1323 & 0.2981 & 0.1211 & 0.5955 & 0.1806 & 0.2635 & 0.3790 & 0.4021 & 0.4235 & 0.4124 & 0.1806 & 0.2635 & 0.3790 \\
\hline SD1ASS & -0.2900 & -0.2818 & -0.0553 & -0.0345 & 0.2485 & 0.0768 & 0.0853 & 0.1620 & 0.0282 & 0.0925 & -0.0436 & 0.0768 & 0.0853 & 0.1620 \\
\hline SD2 & 0.5065 & 0.5168 & 0.4209 & 0.5521 & 0.1105 & 0.2372 & 0.2522 & 0.1280 & 0.1216 & 0.0528 & 0.1039 & 0.2372 & 0.2522 & 0.1280 \\
\hline SD2a_right & 0.4979 & 0.5058 & 0.3696 & 0.5540 & 0.0854 & 0.2048 & 0.2280 & 0.1193 & 0.1216 & 0.0461 & 0.1088 & 0.2048 & 0.2280 & 0.1193 \\
\hline SD2d_left & 0.5155 & 0.5276 & 0.4879 & 0.5526 & 0.1471 & 0.2782 & 0.2866 & 0.1517 & 0.1346 & 0.0753 & 0.1088 & 0.2782 & 0.2866 & 0.1517 \\
\hline SD2a_low & 0.4679 & 0.4651 & 0.4039 & 0.6960 & 0.2856 & 0.1477 & 0.2138 & 0.2022 & 0.2769 & 0.1995 & 0.2440 & 0.1477 & 0.2138 & 0.2022 \\
\hline SD2d_high & 0.5220 & 0.5282 & 0.2621 & 0.3593 & -0.1375 & 0.2368 & 0.2066 & -0.0212 & -0.0656 & -0.1250 & -0.0535 & 0.2368 & 0.2066 & -0.0212 \\
\hline SD2AS & -0.2682 & -0.2858 & 0.0038 & 0.0869 & 0.3049 & 0.0163 & 0.1000 & 0.2831 & 0.4348 & 0.3842 & 0.3601 & 0.0163 & 0.1000 & 0.2831 \\
\hline SD2ASS & -0.3023 & -0.3444 & -0.5357 & -0.2182 & -0.3066 & -0.3944 & -0.3793 & -0.2759 & -0.1571 & -0.2628 & -0.0884 & -0.3944 & -0.3793 & -0.2759 \\
\hline SD1a_right-SD1d_left & -0.1253 & -0.1382 & 0.2995 & 0.0906 & 0.5549 & 0.2871 & 0.3624 & 0.4828 & 0.4612 & 0.4923 & 0.4331 & 0.2871 & 0.3624 & 0.4828 \\
\hline SD2a_low-SD2d_high & -0.0219 & -0.0417 & 0.1961 & 0.2852 & 0.3276 & 0.0031 & 0.1126 & 0.2909 & 0.4609 & 0.4019 & 0.4021 & 0.0031 & 0.1126 & 0.2909 \\
\hline SD1a_low-SD1d_high & -0.2989 & -0.2944 & -0.0299 & -0.0335 & 0.2640 & 0.0454 & 0.0729 & 0.1658 & 0.0368 & 0.1299 & -0.0311 & 0.0454 & 0.0729 & 0.1658 \\
\hline SD2a_right-SD2d_left & -0.2571 & -0.2993 & -0.4386 & -0.1358 & -0.2807 & -0.4497 & -0.3952 & -0.2438 & -0.0780 & -0.1811 & 0.0077 & -0.4497 & -0.3952 & -0.2438 \\
\hline
\end{tabular}


Table 4. Correlation coefficients between HR- and fatigue measures for the entire subject group

\begin{tabular}{|c|c|c|c|c|c|c|c|c|c|c|c|c|c|c|}
\hline \multirow[b]{2}{*}{$\mathrm{p}<0.01$} & \multicolumn{5}{|c|}{ Driving } & \multicolumn{6}{|c|}{ CTT } & \multicolumn{3}{|c|}{ PVT } \\
\hline & MSE & Acc & KSS & LaneDev & PERCL & M Dist & SD Dist & M Velc & SD Velc & M Force & SD Force & M Late & SD Latc & Lapses \\
\hline HR & 0.3560 & 0.0364 & 0.4260 & 0.0250 & 0.5940 & 0.2789 & 0.1925 & -0.1281 & -0.2332 & -0.7108 & -0.3760 & 0.2068 & 0.1867 & -0.3961 \\
\hline HRV & 0.4167 & 0.7408 & 0.3883 & 0.6807 & 0.3345 & 0.6015 & 0.5685 & 0.2931 & 0.1769 & -0.3187 & 0.0170 & 0.6609 & 0.5823 & 0.0292 \\
\hline pNN50 & -0.0069 & 0.0550 & 0.0767 & -0.0027 & 0.2091 & 0.2306 & 0.1525 & -0.0552 & -0.1615 & -0.5386 & -0.2471 & -0.5220 & -0.3656 & 0.0675 \\
\hline ZeroCross & -0.5747 & -0.8394 & -0.5521 & -0.9037 & -0.2909 & -0.7253 & -0.7042 & -0.6550 & -0.5334 & -0.2624 & -0.4319 & -0.7728 & -0.6689 & -0.2249 \\
\hline SD1 & 0.1329 & 0.2129 & 0.1980 & 0.1460 & 0.2223 & 0.2395 & 0.1657 & -0.0954 & -0.1763 & -0.5908 & -0.2548 & -0.5762 & -0.4631 & 0.0301 \\
\hline SD1a_right & 0.2999 & 0.4407 & 0.3455 & 0.3848 & 0.3539 & 0.4446 & 0.3821 & 0.1516 & 0.0432 & -0.4552 & -0.0931 & -0.4049 & -0.3989 & -0.1109 \\
\hline SD1d_left & 0.0781 & 0,1861 & 0.1437 & 0.1222 & 0.0966 & 0.1927 & 0.1184 & -0.1278 & -0.2043 & -0.5559 & -0.2484 & -0.5717 & -0.4768 & 0.0452 \\
\hline SD1a_low & 0.1046 & 0.0543 & 0.1937 & 0.0321 & 0.2730 & 0.0865 & 0.0206 & -0.2210 & -0.3032 & -0.6439 & -0.4471 & -0.5893 & -0.4655 & -0.0448 \\
\hline SD1d_high & 0.1981 & 0.2921 & 0.2580 & 0.2385 & 0.2474 & 0.2057 & 0.1431 & -0.0583 & -0.0828 & -0.4470 & -0.0902 & -0.3796 & -0.3299 & -0.0104 \\
\hline SD1AS & 0.3911 & 0.2839 & 0.3221 & 0.3616 & 0.4301 & 0.4647 & 0.4508 & 0.4937 & 0.3758 & 0.0951 & 0.2276 & 0.0231 & -0.0956 & -0.1461 \\
\hline SD1ASS & -0.3030 & -0.4476 & -0.3294 & -0.4185 & -0.1288 & -0.3359 & -0.3423 & -0.3991 & -0.4069 & -0.2963 & -0.4843 & -0.4697 & -0.3775 & -0.1864 \\
\hline SD2 & 0.4559 & 0.7732 & 0.4283 & 0.7265 & 0.3572 & 0.6359 & 0.6084 & 0.3380 & 0.2259 & -0.2792 & 0.0585 & 0.7181 & 0.6326 & 0.0540 \\
\hline SD2a_right & 0.4437 & 0.7601 & 0.4128 & 0.6991 & 0.3481 & 0.5886 & 0.5599 & 0.2952 & 0.1822 & -0.3106 & 0.0226 & 0.6773 & 0.6094 & 0.1028 \\
\hline SD2d_left & 0.4855 & 0.7897 & 0.4624 & 0.7654 & 0.3821 & 0.6885 & 0.6609 & 0.3896 & 0.2835 & -0.2362 & 0.1074 & 0.7547 & 0.6582 & -0.0206 \\
\hline SD2a_low & 0.4095 & 0.7504 & 0.3382 & 0.7087 & 0.3386 & 0.6695 & 0.6435 & 0.3824 & 0.2939 & -0.2310 & 0.1130 & 0.5180 & 0.4889 & 0.2800 \\
\hline SD2d_high & 0.4897 & 0.6874 & 0.5138 & 0.6585 & 0.3891 & 0.3979 & 0.3662 & 0.1332 & 0.0253 & -0.3154 & -0.0357 & 0.6136 & 0.5806 & 0.0201 \\
\hline SD2AS & -0.3357 & -0.1859 & -0.4682 & -0.2277 & -0.2451 & 0.3186 & 0.2783 & 0.1796 & 0.1092 & -0.1727 & 0.0006 & -0.3570 & -0.4043 & -0.2237 \\
\hline SD2ASS & -0.2134 & -0.4149 & -0.1985 & -0.4913 & 0.0282 & -0.3029 & -0.3575 & -0.3109 & -0.3641 & -0.3930 & -0.2939 & 0.0917 & 0.1075 & 0.2290 \\
\hline SD1a_right-SD1d_left & 0.2196 & 0.1313 & 0.1577 & 0.2247 & 0.2840 & 0.4046 & 0.4045 & 0.4566 & 0.3847 & 0.1373 & 0.2509 & 0.1669 & 0.0238 & -0.1132 \\
\hline SD2a_low-SD2d_high & -0.1187 & 0.0121 & -0.2721 & -0.0328 & -0.0666 & 0.4306 & 0.3815 & 0.1838 & 0.1184 & -0.2789 & -0.0352 & -0.0436 & -0.1331 & -0.1306 \\
\hline SD1a_low-SD1d_high & -0.3182 & -0.4444 & -0.3380 & -0.3931 & -0.2079 & -0.2196 & -0.2396 & -0.2628 & -0.3019 & -0.2394 & -0.4103 & -0.3847 & -0.2799 & 0.0286 \\
\hline SD2a_right-SD2d_left & 0.0115 & -0.2247 & 0.0313 & -0.2837 & 0.2330 & -0.2167 & -0.2602 & -0.2033 & -0.2761 & -0.3291 & -0.1978 & 0.1223 & 0.1300 & 0.1656 \\
\hline
\end{tabular}

Note: Significant values are marked gray

\section{REFERENCES}

Coumel P, Maison-Blanche P, Catuli D (1995) Heart rate and heart rate variability, in Heart rate variability. Malik M, Camm AJ (eds.), Futura Publishing Company, Inc.

Lamond N, Dawson D, Roach GD (2005) Fatigue Assessment in the Field: Validation of a Hand-Held Electronic Psychomotor Vigilance Task. Aviation, Space, and Environmental Medicine, 76.

Levy MN (1977) Parasympathetic control of the heart, in Neural Regulation of the Heart. Randall WC (ed.), Oxford University Press, New York.

Hefner R, Edwards D, Heinze C, Sommer D, Golz M, Sirois B, Trutschel U (2009) Operator Fatigue Estimation Using Heart Rate Measures. Proc $5^{\text {th }}$ Int Driving Symp Human Factors Driver Assessment Training Vehicle Design. Big Sky/Montana, USA, pp 110-117.

Piskorski J, Guzik P (2007) Geometry of the Poincaré plot of RR intervals and its asymmetry in healthy adults. Physiol. Meas., 28, pp 287-300.

Sommer D, Golz M, Schnupp T, Krajewski J, Trutschel U, Edwards D (2009) A Measure of Strong Driver Fatigue. Proc $5^{\text {th }}$ Int Driving Symp Human Factors Driver Assessment Training Vehicle Design, Big Sky/Montana, USA, pp 9-15.

Van Orden KF, Jung TP, Makeig S (2000) Combined eye activity measures accurately estimate changes in sustained visual task performance. Biological Psychology, 52, pp 221-240. 